\title{
DEGRADAÇÃO MECÂNICA DO COQUE EM TESTES DE TAMBOREAMENTO
}

\author{
Antonio Marlon Barros Silva ${ }^{1,2}$ \\ Guilherme Liziero Ruggio da Silva ${ }^{2,3}$ \\ Paulo Santos Assis ' \\ Alfredo Carlos Bitaraes Quintas ${ }^{4}$
}

\section{Resumo}

Esse estudo avaliou a resistência mecânica a frio do coque, utilizando pequenas quantidades ( $300 \mathrm{a} I 200 \mathrm{~g})$ desse material, como alternativa aos testes usuais que utilizam 10 e $50 \mathrm{~kg}$ de coque. Para isso, as degradações volumétrica e superficial foram avaliadas em um tambor de menores dimensões (Diâmetro: $268 \mathrm{~mm}$, comprimento: $470 \mathrm{~mm}$ ), bem como no tambor especificado pela norma JIS K2 I5I (I,5 x I,5 m). Para o equipamento menor encontrou-se que a taxa de degradação superficial $(K)$ aumenta ligeiramente com a massa tamboreada. $O$ fator de maior relevância foi a velocidade de rotação do tambor, que variou de 24 a $85 \mathrm{rpm}$. Nesse caso, $\mathrm{K}$ decresce com a velocidade. Embora a $24 \mathrm{rpm}$ a taxa de degradação superficial tenha sido alta, a quebra volumétrica não ocorreu de forma relevante, sendo que essa ocorreu somente acima de $45 \mathrm{rpm}$. No tambor maior obteve-se uma maior degradação, em comparação com o tambor menor. Entretanto, há uma boa correlação entre a degradação nos dois equipamentos, possibilitando obter funções para se estimar a degradação que seria obtida no tambor maior mediante a degradação observada no tambor menor, ou seja, é possível avaliar a degradação do coque utilizando pequenas quantidades desse material.

Palavras-chave: Degradação mecânica do coque; Teste de tamboreamento; Resistência mecânica a frio do coque.

\section{MECHANICAL DEGRADATION OF COKE IN DRUM TEST}

\begin{abstract}
This study aims to evaluate the coke's cold strength using small sample mass (300 to I200g) in alternative to the usual tests which use 10 to $50 \mathrm{~kg}$ of coke. For this objective the surface and volume breakage were evaluated in a small drum (268 mm in diameter and $470 \mathrm{~mm}$ in length) as well as in the drum specified by the regulation JIS K2I5I ( $1.5 \mathrm{~m}$ in diameter and $\mathrm{I} .5 \mathrm{~m}$ in length). For the small equipment it was found that the surface breakage rate constant (K) slightly increases with the mass tumbled. The most relevant factor was the drum rotation speed that varied from 24 to $85 \mathrm{rpm}$. In this case, $\mathrm{K}$ decreases as speed decreases too. Although at $24 \mathrm{rpm}$ this surface breakage was high, the volume breakage did not occur relevantly, and this happened only above $45 \mathrm{rpm}$. When the tumbling was conducted in a bigger drum ( $1.5 \mathrm{~m} \times 1.5 \mathrm{~m})$, it was obtained a higher degradation, comparing to the smaller drum. However, there is a close relationship between the degradation in both drums, allowing to obtain functions that correlate degradation observed in the smaller drum, that is, it is possible to evaluate the coke's degradation using small quantities of this raw material.
\end{abstract}

Keywords: Mechanical coke breakage; Drum test; Coke's cold strength.

\section{INTRODUÇÃO}

A maior parte da produção de aço no mundo está vinculada à redução de uma carga em alto-forno $(\mathrm{AF}) \mathrm{a}$ coque [I], sendo esse o insumo com maior impacto no custo do ferro gusa [2]. Fatores como a exigência de coque que atenda às demandas cada vez mais restritas do $\mathrm{AF} a$ partir de fontes de menor qualidade, logo mais baratas,

'Rede Temática em Engenharia de Materiais - REDEMAT, Escola de Minas, Universidade Federal de Ouro Preto - UFOP, Ouro Preto, MG, Brasil.

E-mail: amarlonsilva@hotmail.com

${ }^{2}$ Departamento de Metalurgia, Instituto Federal de Minas Gerais - IFMG, Ouro Branco, MG, Brasil.

${ }^{3}$ Gerdau Açominas, Ouro Branco, MG, Brasil.

${ }^{4}$ Instituto Federal de Minas Gerais - IFMG, Ouro Branco, MG, Brasil.

2176-1523 (C) 2016 Associação Brasileira de Metalurgia, Materiais e Mineração. Publicado pela ABM. Este é um artigo de acesso aberto distribuído sob os termos da licença Creative Commons CC BY-NC-ND (Attribution-NonCommercial-NoDerivs) - https:// creativecommons.org/licenses/by-nc-nd/4.0\%. 
têm fomentado diversos estudos na área de coqueificação. Em geral, tais estudos são realizados utilizando-se fornos em escala piloto, que, embora operem com menores custos e riscos, comparando-se com a planta industrial, ainda assim limita muitos estudos, em virtude do preço do equipamento bem como fatores operacionais. A avaliação do coque é feita com diversos padrões, cada um com o intuito de simular uma determinada demanda a que o coque estará submetido. Um dos parâmetros é o DI - Drum Index, que faz inferências sobre a resistência mecânica a frio do coque, com base na degradação desse material em um tambor rotativo (Tabela I). Em condições laboratoriais, a mensuração do $\mathrm{DI}$ dificulta certos estudos relacionados ao processo de coqueificação, uma vez que a quantidade de coque utilizada para tal avaliação é grande (os padrões de maior relevância utilizam 10 e $50 \mathrm{~kg}$ de coque, Tabela I). Segundo Díez et al. [3], a possibilidade de se avaliar a resistência mecânica a frio do coque, com uma pequena quantidade desse material, amplia a possibilidade de estudos utilizando-se fornos em escala laboratorial ou semi-piloto, de menor capacidade, e, portanto menor custo.

A resistência do coque pode ser definida como sendo a habilidade de tal material descer pelo alto-forno sem que haja uma excessiva redução no tamanho e produção de finos, mesmo com as solicitações mecânicas, químicas (reação com $\mathrm{CO}_{2(\mathrm{~g})}$ ) e térmicas no interior do alto-forno [4]. Sharma et al. [5] explicam que a redução no tamanho de partícula de coque e geração de finos reduz a permeabilidade de tal reator.

Segundo Okuyama et al. [6], resultados obtidos a partir de dissecação de altos-fornos mostraram que há uma redução considerável no tamanho médio do coque (cerca de $50 \%$ ), bem como uma redução na resistência a frio desse material à medida que o mesmo desce pelo alto-forno.

$O$ fato do percentual de partículas maiores reduzir e o de finos aumentar na zona granular do alto-forno implica que, nessa parte do reator, a degradação mecânica é predominantemente por abrasão superficial [7] (Figura I).

Os índices de resistência obtidos, em testes de tamboreamento, estão relacionados com a porcentagem de massa que passa e/ou é retida em uma determinada peneira após um certo número de revoluções [9]. Segundo Okuyama et al. [6], o teste de tamboreamento é um método muito útil para a medição da resistência de materiais heterogêneos, como o coque.

Esse tipo de teste reflete com eficiência a habilidade do coque em resistir à degradação antes do carregamento no alto-forno e nas partes superiores desse reator [10]. $E$, embora o teste em um tambor rotativo não simule todas as solicitações a que o coque está submetido em um alto-forno, segundo Okuyama et al. [I I], lotes de coque com baixo desempenho nesses testes dificultam a operação do alto-forno.

Em testes de tamboreamento a fragmentação ocorre por fissuração volumétrica, devido à propagação de trincas preexistentes no material e também degradação superficial, gerando finos [12]. Na Figura I tem-se uma esquematização dos mecanismos de degradação.

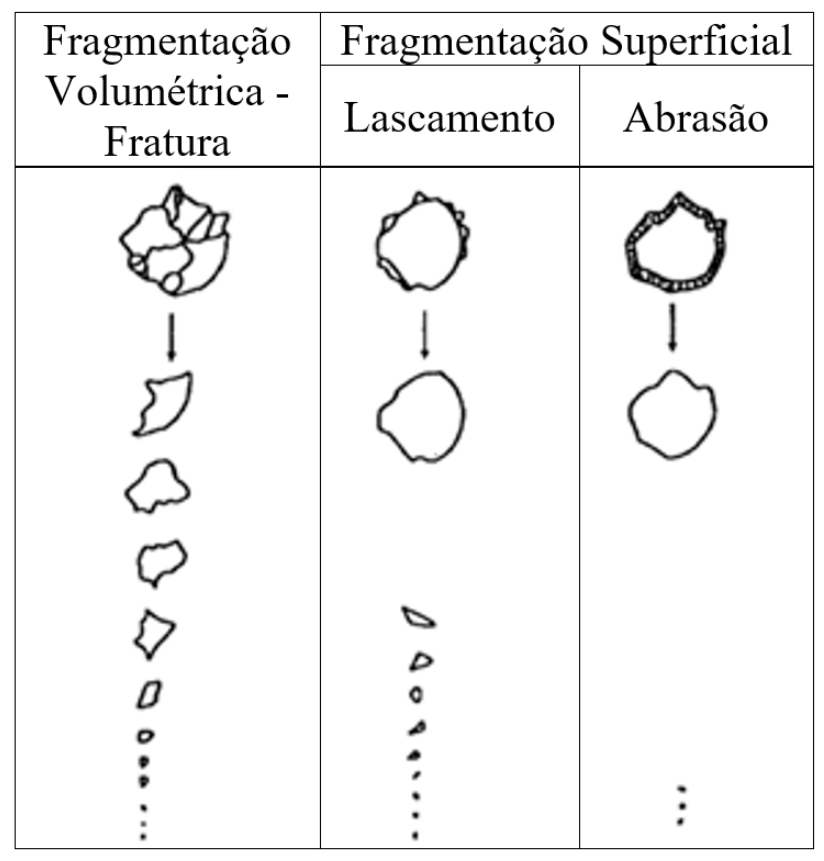

Figura I. Mecanismos de fragmentação. Adaptado de [8].

Tabela I. Padrões de maior relevância para avaliação da resistência mecânica do coque

\begin{tabular}{|c|c|c|c|c|}
\hline Tambor & MICUM & IRSID & JIS & ASTM \\
\hline Norma & M03-046 & M03-046 & K2I5I & D3402 \\
\hline Granulometria do coque (mm) & 60 & 20 & 25 & $+51-76$ \\
\hline Massa da amostra (kg) & 50 & 50 & 10 & 50 \\
\hline Dimensões do tambor (m) & $1,0 \times 1,0$ & $\mathrm{I}, 0 \times \mathrm{I}, 0$ & $\mathrm{I}, 5 \times \mathrm{I}, 5$ & $0,914 \times 0,457$ \\
\hline Velocidade de rotação (rpm) & 25 & 25 & 15 & 24 \\
\hline Total de revoluções & 100 & 500 & 30 ou 150 & 1400 \\
\hline Peneiras (mm) & $60 ; 40$ e 10 & $40 ; 20$ e 10 & $50 ; 25$ e 15 & 25 e 6 \\
\hline \multirow{3}{*}{ Índices } & M40 & 140 & $\mathrm{DI}_{15}{ }^{30} \mathrm{e} \mathrm{DI}_{15}{ }^{150}$ & Estabilidade $(+25 \mathrm{~mm})$ \\
\hline & & & $\%>15$ mm após & \\
\hline & MIO & 120 e 110 & $\begin{array}{l}30 \text { e } 150 \text { revoluções, } \\
\text { respectivamente. }\end{array}$ & Dureza $(+6 \mathrm{~mm})$ \\
\hline
\end{tabular}


Segundo Uchôa [13], as partículas grossas tendem a ser mais frágeis, sendo que, a cada quebra têm-se pedaços de coque cada vez menores e mais resistentes, aumentando-se assim, o grau de estabilização dessa matéria prima.

O grau de estabilização pode ser obtido através do tamboreamento progressivo, no qual é feita uma caracterização granulométrica em intervalos de revolução definidos [13].

\section{METODOLOGIA}

Esse trabalho foi conduzido nas seguintes etapas: (I) - Caracterização física, química, petrográfica e metalúrgica da mistura de carvões submetidos à coqueificação, bem como do coque utilizado, (II) Estudo da degradação mecânica por tamboreamento de coque produzido em uma bateria industrial (comparação entre o tambor industrial, segundo a norma JIS K2 I5I, e um modelo em escala laboratorial).

\section{I Estudo da Degradação Mecânica por Tamboreamento}

coque foi produzido em uma planta industrial (coqueria da Gerdau, Usina Ouro Branco - MG) e teve uma determinação prévia do DI - Drum Index, seguindo a norma JIS K2I5I. Em amostras desse lote de coque, fez-se o estudo da degradação em um tambor de menores dimensões (Diâmetro $268 \mathrm{~mm}$, comprimento $470 \mathrm{~mm}$ ). Para isso, foram realizadas análises granulométricas a cada 100 revoluções no teste de tamboreamento, até um valor de 1200 revoluções. Após 1200 revoluções a distribuição granulométrica foi feita a cada 300 revoluções, totalizando 4200 revoluções. Essa sequência de testes foi realizada em quatro velocidades, 24, 45, 72 e 85 rpm. Para cada velocidade, utilizaram-se três valores de massa, 300, 800 e I $200 \mathrm{~g}$.

Três amostras de coque (300 g), com diferentes faixas granulométricas $(50-37,5 ; 37,5-25,4 ; 25,4-9,5 \mathrm{~mm})$ foram submetidas ao tamboreamento/peneiramento, seguindo a mesma metodologia descrita anteriormente.

Tamboreamento progressivo também foi realizado em um tambor de maiores dimensões ( I,5 m de diâmetro $x$ I,5 m de comprimento, de acordo com a norma JIS K2 I 5 I). Nesse caso, o peneiramento foi realizado a cada 30 revoluções, até atingir-se o valor de 150 revoluções.

\section{RESULTADOS E DISCUSSÃO}

coque utilizado nessa pesquisa foi produzido por uma coqueria do tipo By Product. A caracterização da mistura de carvão que gerou o coque tamboreado, bem como do coque utilizado, está de acordo com a Tabela 2.

Com o tamboreamento a 24 rpm a degradação foi majoritariamente superficial, sendo a fissuração volumétrica irrelevante durante o teste, como se observa na Figura 2.

Para a avaliação da degradação superficial, geração de finos, normalmente aplica-se a malha de I,0 mm [14]. Segundo Litster et al. [14], para esse tipo de degradação, mediante o tamboreamento, é conveniente utilizar a Equação de primeira ordem I:

$$
\frac{d m}{d N}=-K m
$$

\section{Em que:}

$\mathrm{m}$ - Massa remanescente $(>\mathrm{I} \mathrm{mm})$ de material após tamboreamento;

$$
\begin{aligned}
& \mathrm{N} \text { - Número de revoluções (rev.); } \\
& \mathrm{K} \text { - Constante, taxa de degradação superficial }\left(\mathrm{rev}^{-1}\right) \text {. }
\end{aligned}
$$

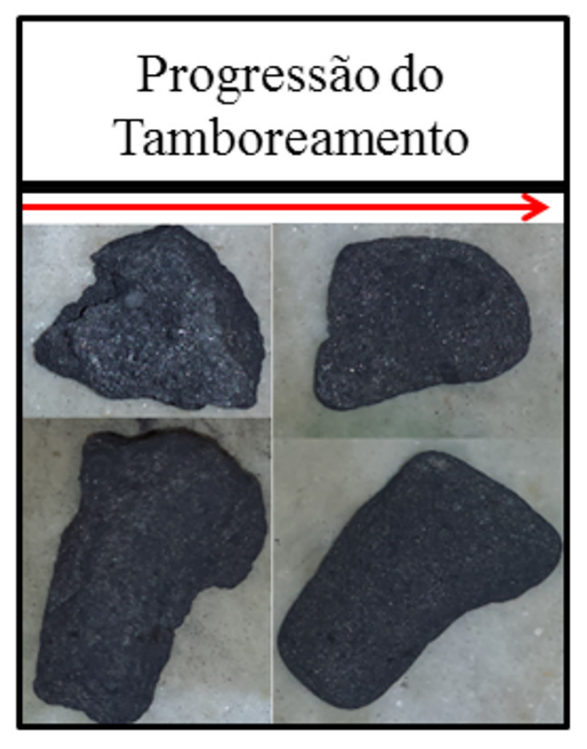

Figura 2. "Polimento" do coque mediante o tamboreamento a

\begin{tabular}{|c|c|c|c|c|c|c|c|c|c|c|c|c|}
\hline \multicolumn{13}{|c|}{ Propriedades } \\
\hline \multirow{2}{*}{$\begin{array}{l}\bar{\pi} \\
\frac{\pi}{2} \\
\frac{\pi}{\pi} \\
\Sigma\end{array}$} & \multirow{2}{*}{ 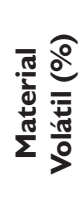 } & \multirow{2}{*}{ 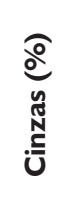 } & \multirow{2}{*}{ 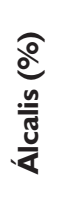 } & \multirow{2}{*}{$\underbrace{\varrho}_{n}$} & \multirow{2}{*}{$\begin{array}{l}\text { } \\
\stackrel{0}{0} \\
\frac{0}{N}\end{array}$} & \multirow{2}{*}{ 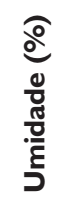 } & \multirow{2}{*}{$\begin{array}{l}\text { № } \\
\frac{0}{0} \\
\frac{D}{3} \text { 믐 }\end{array}$} & \multirow{2}{*}{ 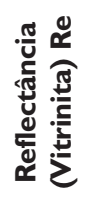 } & \multicolumn{2}{|c|}{$\begin{array}{l}\text { Coque - Tamanho } \\
\text { Médio }(\mathrm{mm})\end{array}$} & \multirow{2}{*}{ 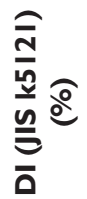 } & \multirow{2}{*}{ 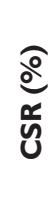 } \\
\hline & & & & & & & & & $\begin{array}{l}\text { Após } \\
\text { Coqueria }\end{array}$ & $\begin{array}{l}\text { Antes } \\
\text { (AF) }\end{array}$ & & \\
\hline Coque & $\mathrm{I}, 2$ & 7,8 & 2,2 & 0,65 & 0,01 & 4,5 & ---- & ----- & 65,0 & 55,0 & 84,5 & 70 \\
\hline $\begin{array}{c}\text { Carvão- Mistura } \\
\text { Base }\end{array}$ & 20,0 & 6,0 & 2,2 & 0,8 & 0,01 & 8,0 & 300 & 1,10 & -------- & ----.. & ----- & ----- \\
\hline
\end{tabular}
24 rpm-4200 revoluções.

Tabela 2. Caracterização química, física, metalúrgica e petrográfica dos insumos testados 
Integrando a equação (I): $\int_{m_{0}}^{m} \frac{d m}{m}=-\int_{0}^{N} K d N$,

tem-se: $\ln \left(\frac{m_{0}}{m}\right)=K N$

Sendo $\mathrm{m}_{0}$ a massa inicial utilizada no teste.

$\mathrm{O}$ valor de $\mathrm{K}$ (taxa de degradação superficial), em rev. $^{-1}$, pode ser obtido pela inclinação da reta $\ln \left(\mathrm{m}_{0} / \mathrm{m}\right)$ vs $\mathrm{N}$, Equação 2. Tal manipulação, para diferentes valores de massa tamboreada e velocidade de rotação, é mostrada na Figura 3 (tambor de laboratório) e na Figura 4 (tambor industrial).

A Equação 2 mostrou-se adequada ao tratamento da degradação superficial, uma vez que se obteve um bom ajuste de curva, sendo tal degradação um processo de primeira ordem. Portanto, com os gráficos da Figura 3, obteve-se o valor de K para diferentes condições de tamboreamento.

Em um tamboreamento progressivo com um tambor de I,5m de diâmetro (norma JIS), obteve-se um valor de $\mathrm{K}$ de aproximadamente $9,0 \times 10^{-4} \mathrm{rev}^{-1}$. Esse valor de Ké da mesma ordem de grandeza $\left(2,7 \times 10^{-4} \mathrm{rev}^{-1}\right)$ do valor encontrado por Litster et al. [9], que utilizou um tambor de I,0 m de diâmetro.

No presente estudo, o valor de $\mathrm{K}$ foi da ordem de $10^{-5}\left(\right.$ rev. $\left.^{-1}\right)$. Tal grandeza tem relação com o diâmetro do tambor (altura de queda).

Embora todas as curvas (Figura 3) tenham obtido alto valor de $R^{2}(0,988-0,997)$, é nítido que a inclinação de uma reta formada pelos primeiros pontos, início do teste de tamboreamento, possui maior inclinação do que uma reta formada pelos pontos no final desse teste. Obtendo-se o

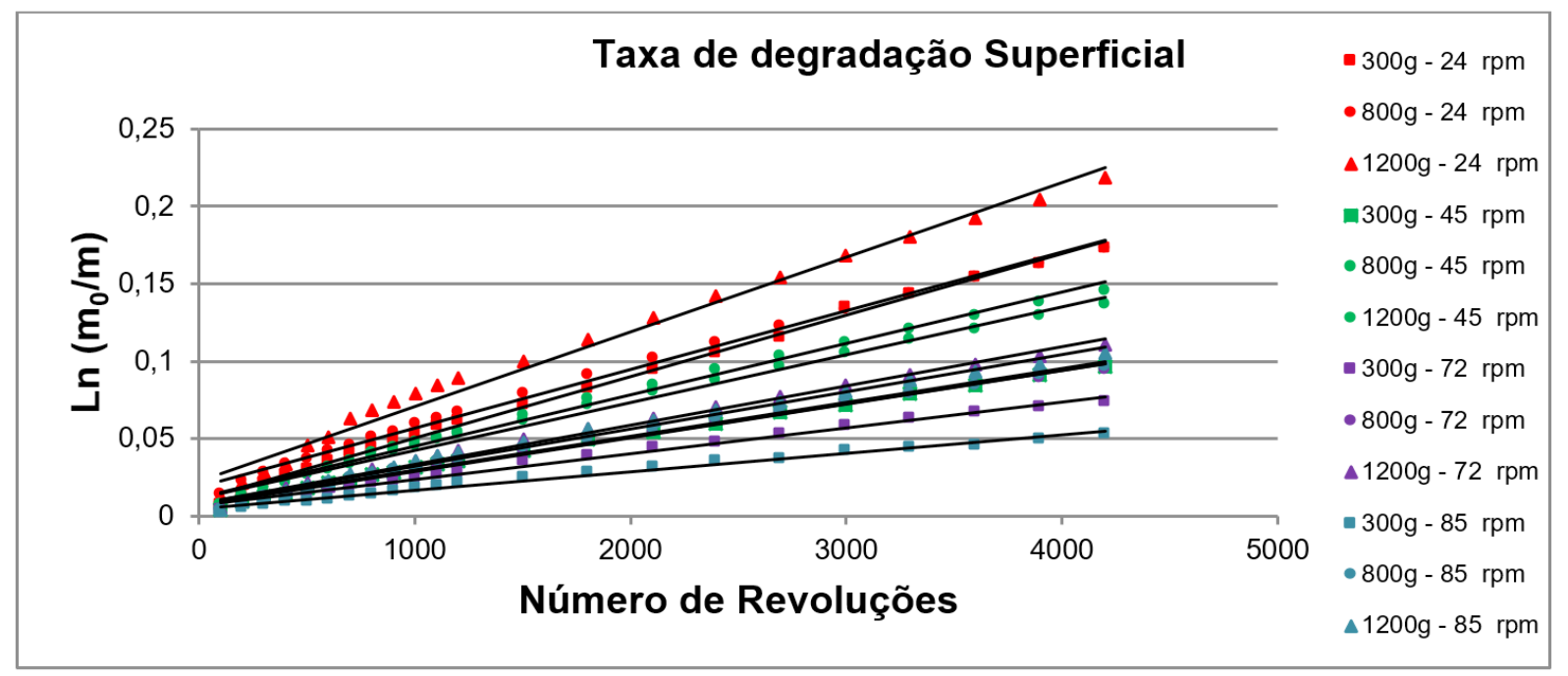

Figura 3. Determinação da Taxa de degradação superficial sob diferentes condições de tamboreamento.

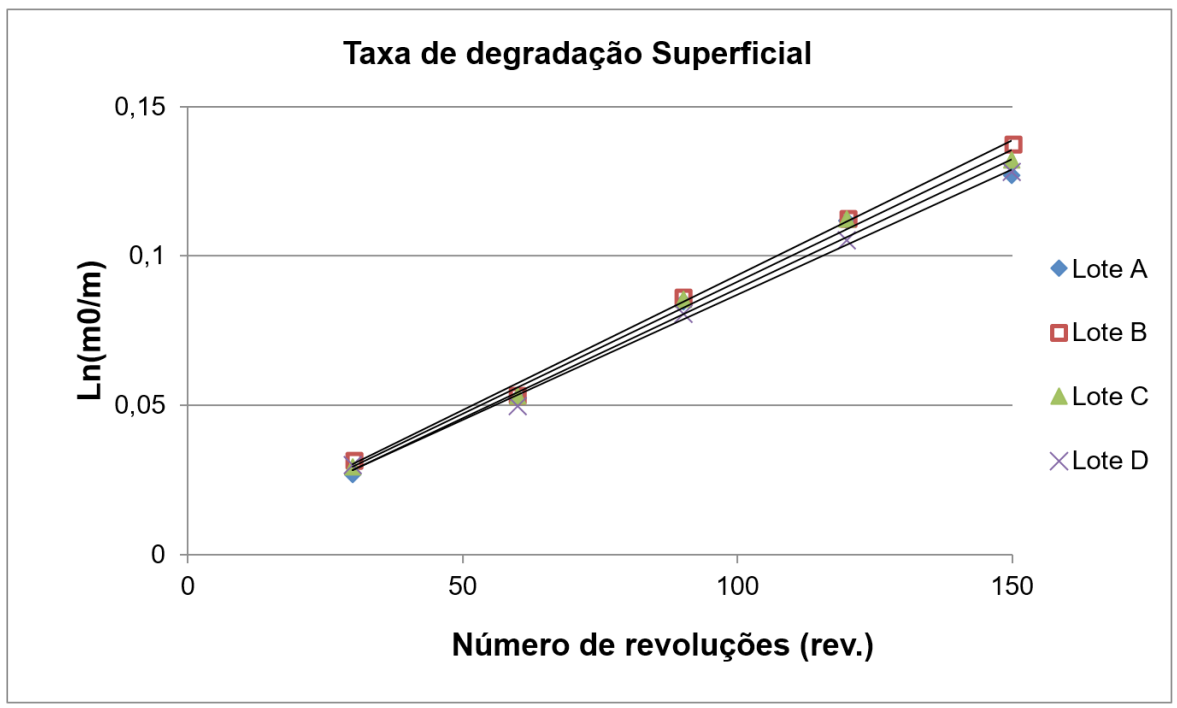

Figura 4. Determinação da Taxa de degradação superficial para o tambor industrial. 
valor de K para cada ponto (segundo a equação no canto superior da Figura 5), tem-se que o valor dessa grandeza varia com o número de rotações conforme o exposto no gráfico da Figura 5.

Inicialmente a taxa de degradação superficial é elevada, reduzindo-se com a progressão do teste. Após cerca de 2000 revoluções, o valor de $\mathrm{K}$ tende a um valor constante. Normalmente aplica-se o conceito de estabilização mecânica de acordo com a fissuração volumétrica do coque, entretanto, pelo exposto na Figura 5, o conceito pode ser estendido à degradação superficial.
Embora não seja atingido um patamar, há uma situação próxima disso.

Segundo Ragan e Marsh [12], é de extrema relevância para o teste de tamboreamento as condições de operação do tambor rotativo. Esse fato fica evidente na Figura 6, na qual se tem a relação de $\mathrm{K}$ com a velocidade de rotação do tambor para diferentes quantidades de coque tamboreada.

À medida que a velocidade aumenta, tem-se maior predominância do regime em catarata, o qual favorece a degradação por impacto, dessa forma a geração de finos é reduzida, por isso há uma redução da constante de

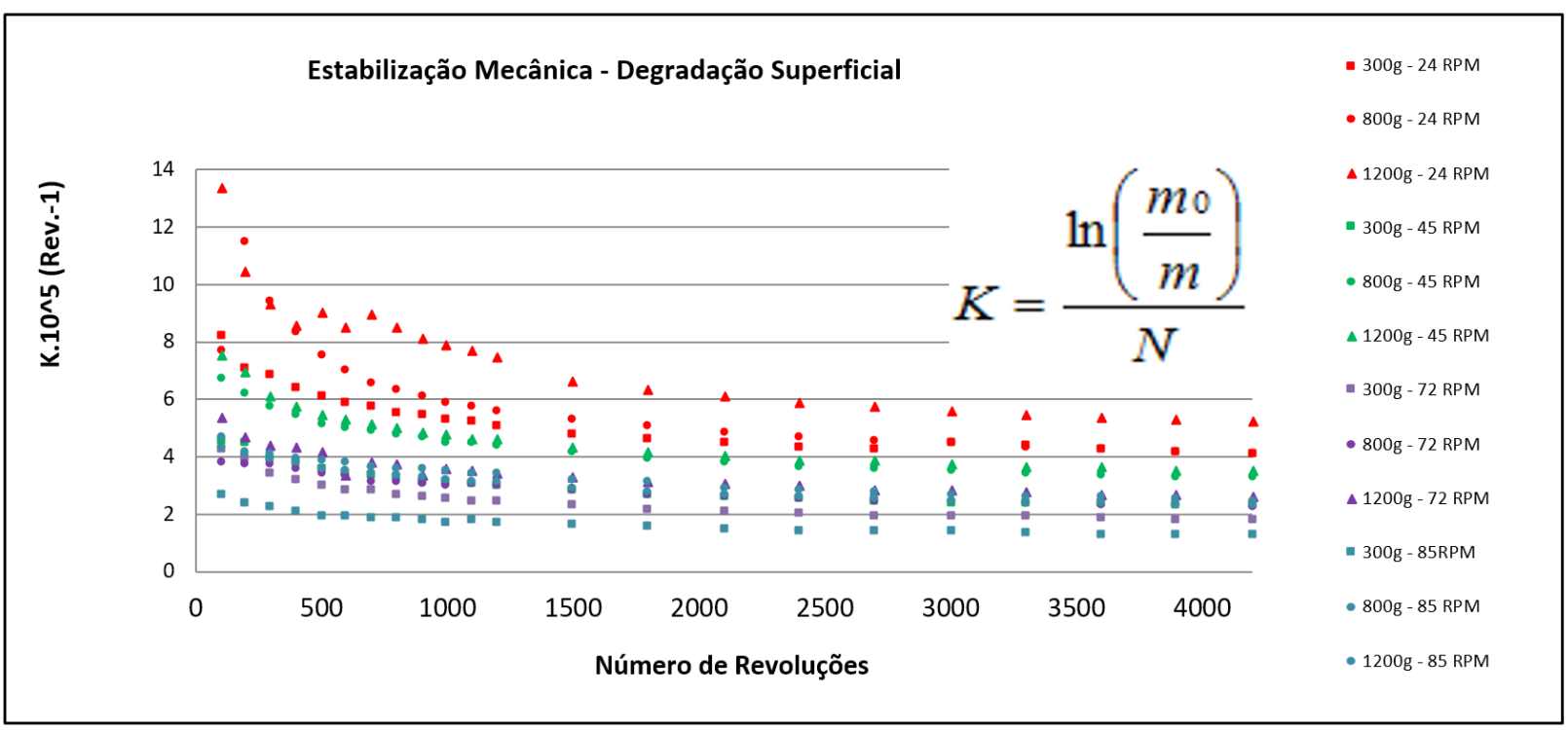

Figura 5. Variação de K com a progressão do tamboreamento (estabilização mecânica).

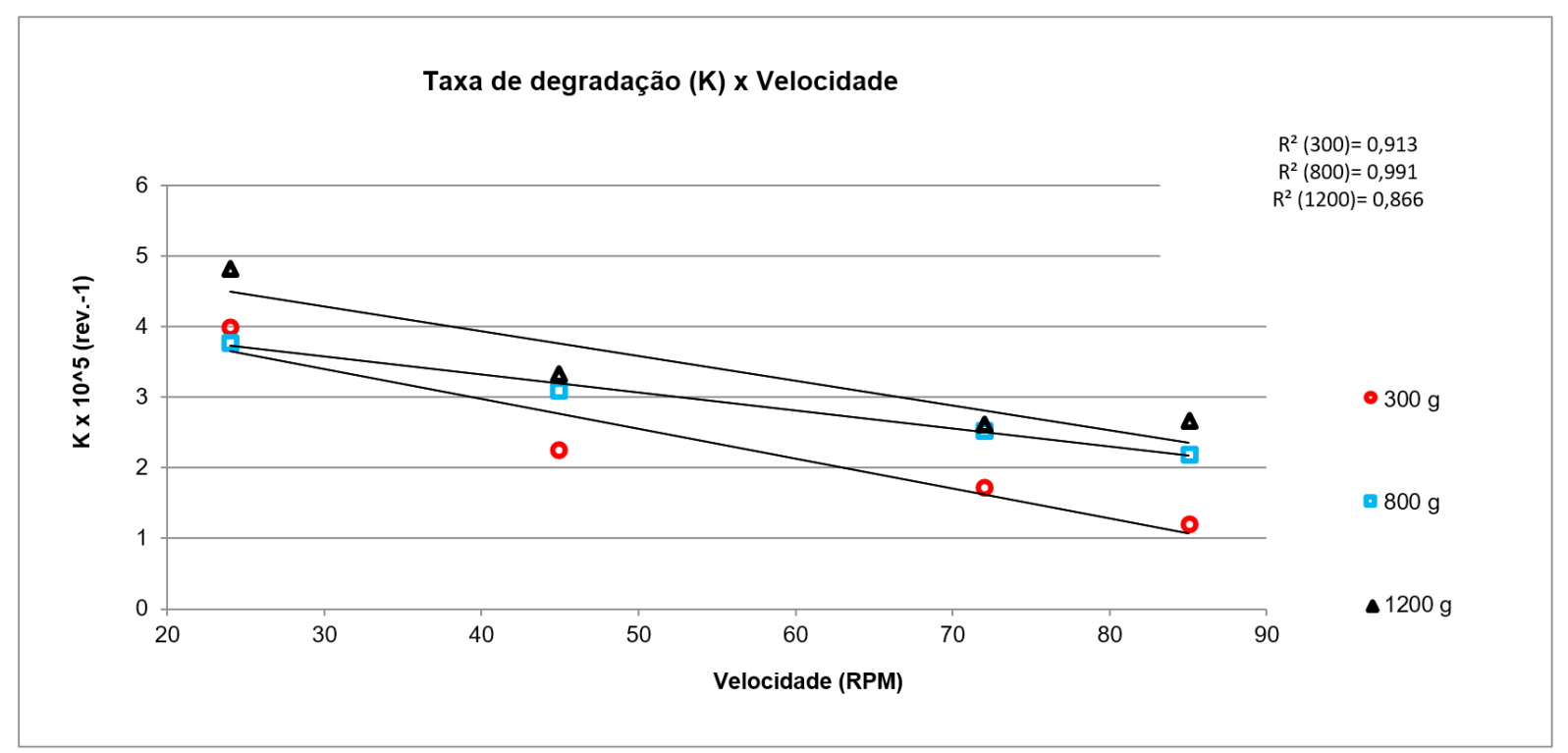

Figura 6. Variação de $\mathrm{K}$ com a velocidade de rotação. 
degradação superficial e aumento na degradação volumétrica. Isso está de acordo com o observado por Litster et al. [9] e Elber et al. [15].

Litster et al. [9], embora tenha utilizado um tambor de maiores dimensões ( 1,0 m de diâmetro) encontrou uma relação próxima à verificada nesse estudo, acima de 25 rpm a taxa de degradação superficial diminui.

Com relação à massa, Litster et al. [9] mostrou que esta é irrelevante para a determinação da taxa de degradação superficial, entretanto seus estudos foram conduzidos utilizando-se massas relativamente altas (acima de $5 \mathrm{~kg}$ ). $\mathrm{Na}$ atual pesquisa, em que foram utilizadas pequenas quantidades de coque, a massa não se mostrou um fator completamente irrelevante durante o teste, uma vez que a degradação tende a aumentar ligeiramente à medida que se aumenta a massa tamboreada, evidenciando-se assim a importância da interação partícula-partícula na degradação do coque.

À medida que o coque utilizado no tamboreamento é de maior tamanho, há uma redução na taxa de degradação superficial, ou seja, a geração de finos é menor (Figura 7). A geração de finos é um fenômeno predominantemente superficial, sendo que, para a mesma massa, maior a área superficial total (menor tamanho de partícula) maior a degradação superficial.

Litster et al. [9] encontrou o inverso de tal observação, entretanto, no estudo desse autor, as partículas de menor granulometria foram obtidas por um tamboreamento prévio até se atingir o tamanho desejado, sendo que, as partículas menores passaram, em geral, por um pré-tamboreamento de maior duração, em comparação com as partículas maiores, antes de se iniciar o teste. Nesse caso as partículas menores tendem a ter maior grau de estabilização. No caso desse estudo não foi realizado tal pré-tamboreamento.

Para quantificar e separar os dois fenômenos de fragmentação (volumétrica e superficial) novos índices foram criados. Tais índices são: DI37, DI25 e DI9, sendo esses correspondentes ao percentual retido nas peneiras acima de
37,5, 25,4 e 9,5 mm, respectivamente. Esses índices visam quantificar a fragmentação volumétrica após o tamboreamento. Para a avaliação da fragmentação superficial (geração de finos) utilizou-se, além do K, o FI (fine index), sendo esse o percentual abaixo de I,0 mm, após o tamboreamento.

Esses novos índices foram utilizados para avaliar e comparar a degradação nos tambores de laboratório e industrial. Para tal, quatro lotes de coque foram utilizados (Lotes A; B; C e D, granulometria: 50,0-37,5 mm, provenientes da coqueria da Gerdau, usina de Ouro Branco-MG). Para o tambor menor, utilizou-se uma velocidade de rotação de 85 rpm, massa de coque de $800 \mathrm{~g}$ e um total de revoluções de 4200. Essa escolha foi baseada no melhor ajuste de curva para o \%retido versus o número de revoluções e que são valores facilmente empregados em laboratório.

Embora a fissuração volumétrica no tambor industrial (Gerdau) tenha sido mais intensa, com o ajuste de curva obtido, plotando-se o \%retido vs o número de rotações, tanto para o tamboreamento na condição laboratorial quanto industrial, foi possível obter funções para se estimar ○\% retido no tambor industrial a partir desses respectivos valores obtidos no tambor menor (Figura 8).

No tamboreamento progressivo do teste industrial peneirou-se o coque em intervalos de 30 revoluções (20\% do número total de revoluções). No caso do tamboreamento de laboratório, utilizou-se um número maior de revoluções, 4200. Aplicando-se intervalos de revolução de $20 \%$, no caso do tamboreamento de laboratório, têm-se os valores correspondentes de número de revolução conforme a Tabela 3. Os intervalos de revolução praticados no tamboreamento progressivo em laboratório não coincidem com os intervalos de $20 \%$ correspondentes ao teste industrial. Portanto, os valores do percentual retido em uma determinada malha (DI) para os valores de "Número de revolução correspondente" foram obtidos mediante interpolação, sem prejuízo na qualidade dos dados, como pode ser observado na Tabela 3.

A mesma metodologia, descrita na Tabela 3, foi aplicada para os percentuais retidos nas malhas de

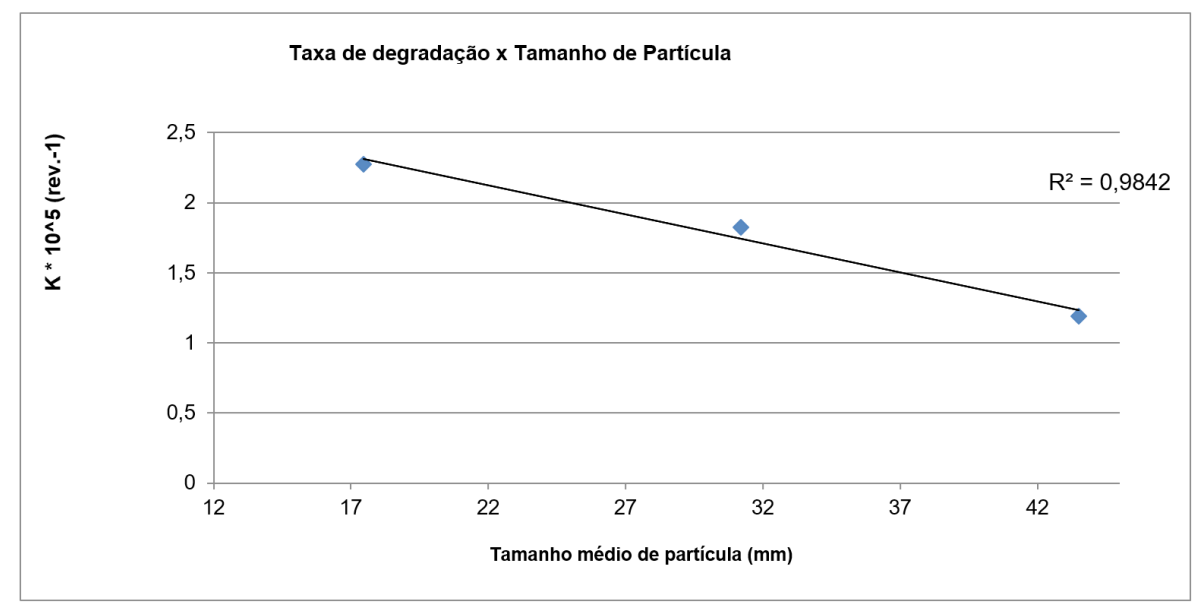

Figura 7. Variação de $\mathrm{K}$ com a velocidade de rotação. 
37,5 25,4 e 9,5 mm, para os quatro lotes, A, B, C e D, o que possibilitou a obtenção das funções para a estimativa dos novos índices, conforme Tabela 4.

$O$ índice de finos (FI) tanto para o tamboreamento com o equipamento industrial quanto com o tambor menor (laboratório) são valores próximos. Por esse motivo, não há necessidade de se estimar tal índice (Tabela 5). Com isso, demonstra-se que o Fl é mais adequado à quantificação da fragmentação superficial (geração de finos) do que a constate de degradação superficial (K) proposta inicialmente.
Como pode ser visto na Figura 8 e na Tabela 5, é possível estimar com boa precisão o percentual retido nas malhas de 25,4 e 9,5 mm no tamboreamento industrial, a partir dos respectivos valores obtidos utilizando o tambor menor. Para o percentual retido na malha de $37,5 \mathrm{~mm}$, não há uma boa estimativa dos valores obtidos com o tamboreamento industrial. Embora os valores médios (estimado e real) sejam próximos, há uma dispersão alta dos resultados (Tabela 5). Essa dispersão pode está associada ao fato de que, embora a faixa granulométrica seja a mesma para os
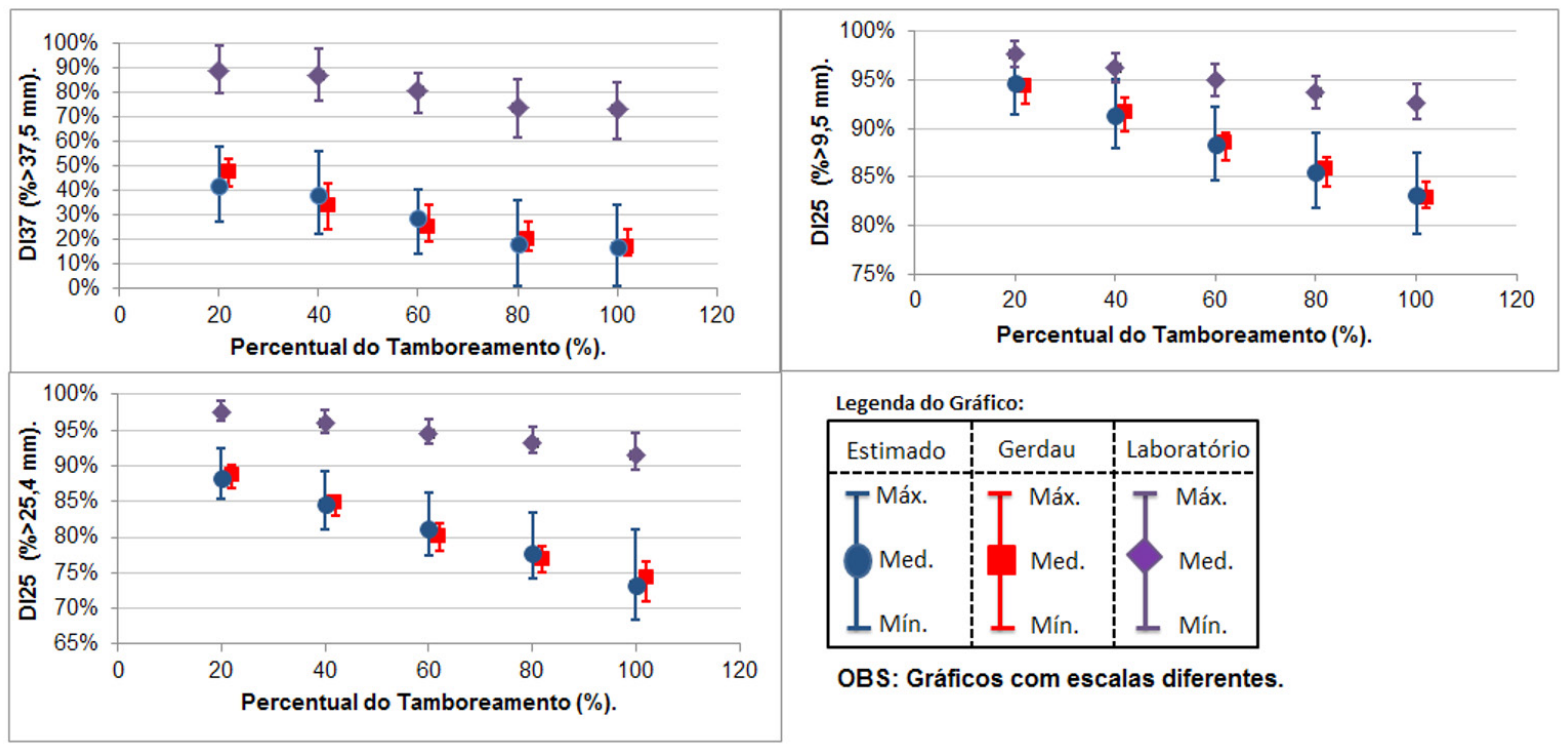

OBS: Gráficos com escalas diferentes.

Figura 8. Porcentagem acima de 37,5, 25,4 e 9,5 mm em tamboreamento progressivo (industrial e laboratorial), bem como a estimativa para os valores obtidos no tamboreamento industrial.

Tabela 3. Metodologia para obtenção de funções que correlacionam o \% retido após tamboreamento no tambor industrial a partir do \% retido após o tamboreamento no tambor menor (laboratório)

\begin{tabular}{|c|c|c|c|c|c|}
\hline $\begin{array}{c}N^{\circ} \text { de revoluções- } \\
\text { teste Industrial. JIS } \\
\text { K 2I5 I }\end{array}$ & $\%$ Teste & $\begin{array}{l}\text { No de revoluções } \\
\text { correspondentes- } \\
\text { escala laboratorial }\end{array}$ & $\begin{array}{l}\text { No de revoluções } \\
\text { realizadas - escala } \\
\text { de Laboratório }\end{array}$ & $\begin{array}{c}\%>25,4 \\
\text { Laboratório (Média } \\
\text { dos } 4 \text { lotes) }\end{array}$ & $\begin{array}{c}\%>25,4 \text { para } n^{\circ} \\
\text { de revoluções } \\
\text { correspondentes - } \\
\text { escala laboratorial } \\
\text { (Interpolação). }\end{array}$ \\
\hline \multirow{2}{*}{30} & \multirow{2}{*}{20} & \multirow{2}{*}{$4200 * 0,2=840$} & 800 & 97,31 & \multirow{2}{*}{97,23} \\
\hline & & & 900 & 97,10 & \\
\hline \multirow{2}{*}{60} & \multirow{2}{*}{40} & \multirow{2}{*}{$4200 * 0,4=1680$} & 1500 & 94,53 & \multirow{2}{*}{94,30} \\
\hline & & & 1800 & 93,96 & \\
\hline \multirow{2}{*}{90} & \multirow{2}{*}{60} & \multirow{2}{*}{$4200 * 0,6=2520$} & 2400 & 92,97 & \multirow{2}{*}{92,78} \\
\hline & & & 2700 & 92,48 & \\
\hline \multirow{2}{*}{120} & \multirow{2}{*}{80} & \multirow{2}{*}{$4200 * 0,8=3360$} & 3300 & 91,68 & \multirow{2}{*}{91,53} \\
\hline & & & 3600 & 91,30 & \\
\hline 150 & 100 & $4200 * 1,0=4200$ & 4200 & 90,50 & 90,50 \\
\hline
\end{tabular}

Tabela 4. Funções obtidas utilizando a metodologia descrita na Tabela 3

\begin{tabular}{cccc}
\hline \multirow{2}{*}{ Valor a Ser Calculado $-\mathbf{F}(\mathbf{x})$} & $\mathbf{X}$ & \multicolumn{2}{c}{$\mathbf{F u n c ̧ a ̃ o ~} \mathbf{F}(\mathbf{x}) \mathbf{=}$ ax $+\mathbf{b}$} \\
\cline { 3 - 4 }$\%>37.5$ Gerdau & $\%>37,5$ (Lab.) & 1,596 & $\mathbf{b}$ \\
$\%>25.4$ Gerdau & $\%>25,4$ (Lab.) & 2,496 & $-0,999$ \\
$\%>9.5$ Gerdau & $\%>9,5$ (Lab.) & 2,304 & $-1,549$ \\
\hline
\end{tabular}


Tabela 5. Índices de fissuração volumétrica e superficial

\begin{tabular}{|c|c|c|c|c|c|c|c|c|c|c|c|}
\hline \multirow[b]{2}{*}{ ֻّ } & \multicolumn{3}{|c|}{$\%>37,5 \mathrm{~mm}$} & \multicolumn{3}{|c|}{$\%>25,5 \mathrm{~mm}$} & \multicolumn{3}{|c|}{$\%>9,5 \mathrm{~mm}$} & \multicolumn{2}{|c|}{$\%>\mathrm{I}, 0 \mathrm{~mm}(\mathrm{FI})$} \\
\hline & 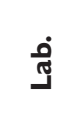 & $\begin{array}{l}\text { J } \\
\frac{\pi}{0} \\
\frac{0}{d} \\
ن\end{array}$ & 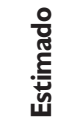 & 官 & 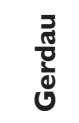 & $\begin{array}{l}\text { 윯 } \\
\text { है } \\
\text { 㞫 }\end{array}$ & ก & 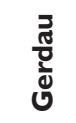 & 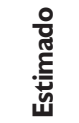 & 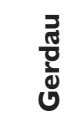 & กี \\
\hline $\mathbf{A}$ & 84,1 & 13,5 & 34,2 & 89,5 & 76,3 & 68,5 & 90,9 & 84,5 & 79,1 & 8,19 & 8,85 \\
\hline B & $6 I, 2$ & 16,0 & 0,0 & 90,5 & 76,6 & 71,0 & 91,7 & 81,8 & 80,8 & 8,02 & 8,04 \\
\hline C & 65,5 & 14,4 & 4,6 & 91,2 & 71,0 & 72,6 & 93,6 & 83,0 & 85,4 & 7,78 & 8,07 \\
\hline D & 81,5 & 24,0 & 30,1 & 94,5 & 73,1 & 81,1 & 94,5 & 82,0 & 87,4 & 8,13 & 8,20 \\
\hline Média & 73,1 & 17,0 & 17,2 & 91,4 & 74,3 & 73,3 & 92,7 & 82,8 & 83,2 & 8,03 & 8,29 \\
\hline
\end{tabular}

quatro lotes, não há uma garantia de que o tamanho inicial carregado seja o mesmo. Podendo um lote está próximo ao limite superior $(50,0 \mathrm{~mm})$ e o outro mais próximo ao limite inferior $(37,5 \mathrm{~mm})$.

\section{CONCLUSÕES}

A taxa de degradação superficial é menor à medida que se aumenta a velocidade, sendo que a $24 \mathrm{rpm}$ tem-se a máxima degradação superficial. Já a fissuração volumétrica ocorreu com relevância apenas para as velocidades acima de 45 rpm, sendo que, a 24 rpm a degradação foi praticamente superficial. Isso é devido ao regime de movimentação do material no tambor, que é função da velocidade.

O tamanho de partícula influencia a taxa de degradação superficial, sendo que essa decresce com o tamanho de partícula, ou seja, coques em menores granulometrias geram uma quantidade maior de finos.

Foram obtidas as melhores condições para se testar o coque, utilizando-se para isso pequenas quantidades desse material. Essas condições foram selecionadas comparando-se a progressão do \% retido nas malhas de 37,5; 25,4 e 9,5 mm, tanto no tamboreamento industrial quanto de laboratório, com o número de revoluções.

Existe uma boa correlação entre o percentual retido nas peneiras de 25,4 e 9,5 mm no tamboreamento industrial com o de laboratório, tornando possível quantificar a quebra volumétrica que seria observada no tambor industrial a partir do que é observado no tambor de laboratório. No caso do percentual acima de $37,5 \mathrm{~mm}$ há uma boa estimativa apenas quando são considerados os valores médios (estimado e real)

$\mathrm{O} F \mathrm{Fl}$ (índice de finos) mostrou-se mais adequado do que a constante de degradação superficial $(K)$ para a quantificação da geração de finos, uma vez que há uma correspondência nesse índice utilizando-se os dois equipamentos (industrial e de laboratório). Esse comportamento não foi observado com K, que, no tambor industrial foi cerca de 10 vezes maior do que o encontrado no tamboreamento de laboratório.

\section{Agradecimentos}

Os autores agradecem ao IFMG, campus Ouro Branco - MG, Gerdau, unidade Ouro Branco - Açominas e à REDEMAT/UFOP - Rede Temática em Engenharia de Materiais.

\section{REFERÊNCIAS}

I World Steel Association [página da internet]. Bruxelas. [acesso em 25 mar. 20 I6]. Disponível em: https://www. worldsteel.org/statistics/crude-steel-production-20 I6-20I5.html.

2 Coelho RJ, Silva OJ, Alves MT, Andrade LF, Assis PS. Modelos de previsão da qualidade metalúrgica do coque a partir da qualidade dos carvões individuais. REM - Revista Escola de Minas. 2004;57(I):27-32.

3 Díez MA, Álvarez R, Radoslavov N, Barriocanal C, Canga CS, Xiberta J. Desarrollo de um nuevo método a escala de laboratório para determinar la resistencia mecânica del coque. Revista de Metalurgia. 2002;38:4l 0-4l 8.

4 Curran J. Fundamental factors influencing coke strength [dissertação de mestrado]. Australia: The University of New Castle, 2009.

5 Sharma R, Dharmendra K, Tiwari HP, Banerjee PK. Study on coke size degradation from coke plant wharf to blast furnaces stock house. Koks i Khimiya. 2013; I I:32-39.

6 Okuyama Y, Miayazu T, Kishimoto S. Physical degradation of coke in blast furnace. Transactions ISIJ. 1984;25:302310.

7 Iwanaga Y, Takatani K. Degradation behavior of coke in blast furnace at high-temperature zone. Transactions ISIJ. 1988;28:990-998. 
8 Sahoo R. Degradation characteristics of steel making materials during handling. Powder Technology. 2007; 176:77-87.

9 Litster JD, Waters AG, Nicol SK. A quantitative measure of coke abrasion. Transactions ISIJ. 1986;27:704-709.

I0 Sundholm JL, Valia HS, Kiessling FJ, Richardson J, Buss WE, Worberg R, et al. Manufacture of metallurgical coke and recovery of coal chemicals. In: Fruehan RJ. The making, shaping and treating of steel. II. ed. Pittsburgh: AISE Steel Foundation; 1999. p. 38I-545. (Ironmaking volume).

I I Okuyama Y, Miayazu T, Kishimoto S. Termal degradation of coke in blast furnace. Fuel. 1985;64:475-480.

12 Ragan S, Marsh H. A critique of industrial methods of measurement. Journal of Physics. D, Applied Physics. 1979;13:983-993.

13 Ulhôa MB. Carvão aplicado à fabricação de coque de alto-forno [curso interno]. Ipatinga: Usiminas; 201 I .

I 4 Litster JD, Waters AG, Nicol SK. Coke degradation by surface breakage in a modified tumble drum. Transactions ISIJ. 1987;27:3-9.

15 Elber L, Thomson D, Johnson G. Development of a control system for a sag-b-c grinding circuit. Minerals Engineering. 1994;7:153-167.

Recebido em: 24 Ago 2016

Aceito em: 16 Nov 2016 